\title{
COLLIDING WINDS IN SYMBIOTIC SYSTEMS
}

\author{
Sun Kwok \\ Department of Physics \\ University of Calgary \\ Calgary, Alberta \\ Canada T2N 1 N4
}

\begin{abstract}
The physics of colliding winds in symbiotic systems is reviewed. The theoretical predictions are compared with observational data of symbiotic novae, in particular the recently erupted system HM Sge. It is suggested that the spectral behaviour of HM Sge from X-ray to radio can be explained by the colliding winds process.
\end{abstract}

\section{INTRODUCTION}

One of the most interesting aspects of symbiotic stars is the extreme difference in spectral types between the two components of the system and symbiotic novae represent the most extreme case among symbiotic stars. It is now generally believed that a symbiotic nova system consists of a Mira Variable of effective temperature $\left(\mathrm{T}_{e}\right)<3000 \mathrm{~K}$ and a degenerate white dwarf (probably a $\mathrm{C}-0$ white dwarf) of $\mathrm{T}_{e}>100000 \mathrm{~K}$. While the combination of the large extent of the atmosphere of the cool component and the strong gravity of the hot component suggests the possibility of interaction, in fact the symbiotic novae systems have very large binary separations and very long periods and belong to an evolution scenario more extreme than the Case C class of Paczyǹski (1980). If the outbursts observed in these systems are the result of binary interaction, then stellar winds must play an important role.

Some symbiotic stars are known to contain Mira Variables, which as a class is believed to have large mass loss rates of $>10^{-6} \mathrm{M}_{\odot} \mathrm{yr}^{-1}$. Other symbiotic stars have outbursts similar to that of classical novae which undergo ejections with velocities $>1000 \mathrm{~km} \mathrm{~s}^{-1}$. If both of these physical processes take place within a single system, then the interaction between the two ejecta is inevitable, resulting in physical consequences which manifest in many parts of the electromagnetic spectrum. In this review, we will examine the physics of wind interactions and compare the theoretical predictions with observations.

\section{SYMBIOTIC NOVAE}

The class of objects called symbiotic novae is defined by Allen (1980). 
Among the seven (or eight if PU Vul is included) objects in this class, HM Sge is the one example which has the largest amount of multi-frequency data available. HM Sge has been detected in almost every spectral band from X-ray to radio (Kwok 1982) and has been monitored extensively since its outburst in 1975. Although one can argue that every symbiotic object is unique and it is unfair to concentrate on one object as an representative of the whole class, yet the wealth of data for HM Sge makes it attractive to use this object as a proto-type for the physical phenomenon of colliding winds.

\subsection{The cool component}

The detection of photospheric absorption features of $\mathrm{CO}$ and $\mathrm{H}_{2} \mathrm{O}$ suggests that the cool component in HM Sge is a Mira Variable (Puetter et al. 1978), and this is confirmed by the Mira-like variations due to atmospheric pulsation (Ipatov, Taranova and Yudin 1985). A period of 540 days has been determined by Whitelock (this volume), suggesting that it is a very evolved star on the asymptotic giant branch (AGB). Figure 1 shows the 8-100 $\mu \mathrm{m}$ infrared spectrum of HM Sge as observed by the IRAS satellite. The strong emission features at 10 and $18 \mu \mathrm{m}$ are identical

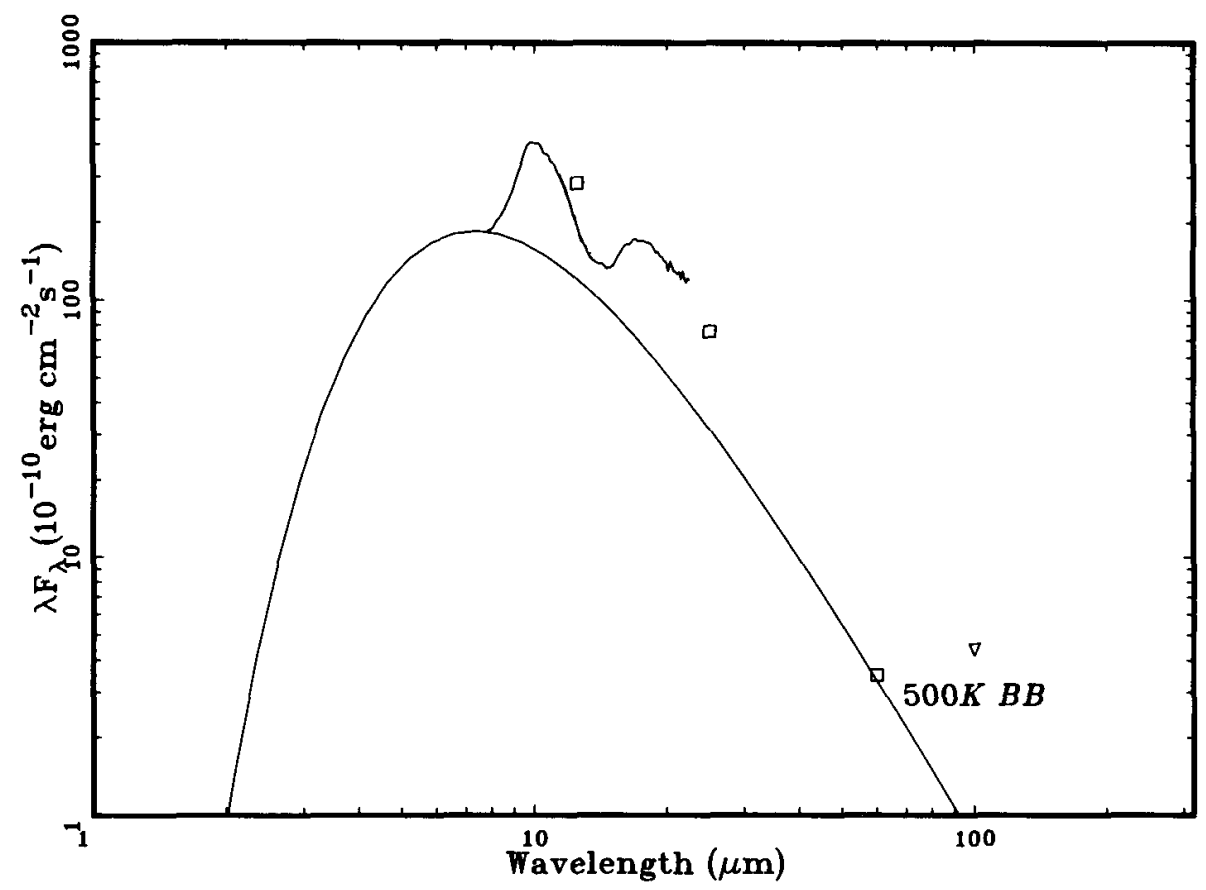

Fig. 1 The IRAS spectrum of HM Sge. The 8-23 $\mu \mathrm{m}$ spectrum is obtained by the Low Resolution Spectrometer and has been normalized to the $12 \mu \mathrm{m}$ photometry point. The four photometric measurements have been corrected for colour. A $500 \mathrm{~K}$ blackbody is also shown for comparison. 
to the circumstellar silicate dust features observed in $>2000$ AGB stars observed by IRAS (Volk and Kwok 1987). This suggests that HM Sge must have a very extended circumstellar envelope which has not been severely disturbed by the outburst event in 1975 .

\subsection{The hot component}

Even in the ultraviolet, the continuum emission is dominated by the nebular component and the existence of the hot component is only inferred from the effect of its ionizing radiation on the circumstellar region. Analysis of the emission lines implies a hot-star temperature of $50,000 \mathrm{~K}$ after the outburst and a temperature of $-160,000 \mathrm{~K}$ now (Stauffer 1984; Mueller and Nussbaumer 1985). The most interesting aspect of the hot star is the detection of a Wolf-Rayet feature suggesting an expansion velocity of $>2000 \mathrm{~km} \mathrm{~s}^{-1}$. The presence of the WR feature is similar to those observed in central stars of planetary nebulae, which are now commonly observed by IUE to have high-velocity mass outflows. Unfortunately the WR phase in HM Sge ended before IUE spectra could be obtained.

\subsection{The nebular component}

The detection of thermal radio emission implies that the ionized nebula extends over a radius $>10^{15} \mathrm{~cm}$. The radio spectrum is optically thick to at least $22 \mathrm{GHz}$, and as in the case of the optically thick phase of classical novae, the flux level has been steadily increasing at al1 wavelengths. In contrast to classical novae, which optically thick phase only lasts a few months, HM Sge has remained optically thick for over 10 years! If we approximate this behaviour by an expanding blackbody then the expansion velocity of the radioemitting region can be estimated to be -56 (D/kpc) $\mathrm{km} \mathrm{s}^{-1}$ (Kwok, Bignell and Purton 1984).

Figure 2 shows the radio light curves of HM Sge as the result of monitoring programs at the Algonquin Radio Observatory and the Very Large Array since 1977. Wallerstein (1978) was the first to note the existence of multiemission components in HM Sge.

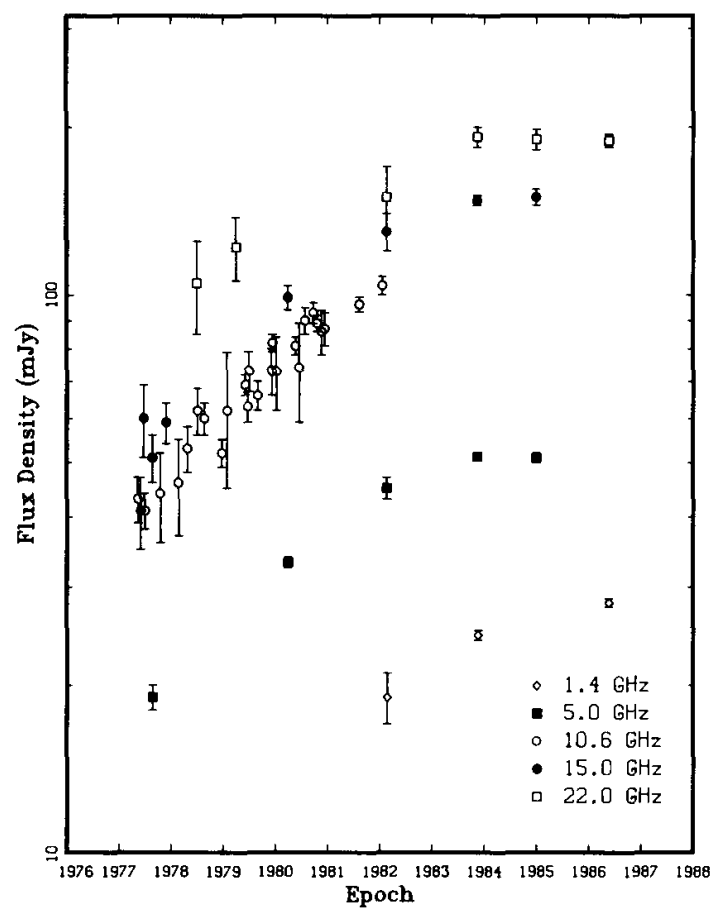

Fig. 2 Radio light curves of HM Sge. 
He found that while permitted lines like $\mathrm{H} \alpha$ and HeI have broad wings indicative of expansion velocities of $1700 \mathrm{~km} \mathrm{~s}^{-1}$, [OI] and [SIII] show velocities of $75 \mathrm{~km} \mathrm{~s}^{-1}$ and [NII] is very narrow with a velocity of 20 $\mathrm{km} \mathrm{s}^{-1}$.

\section{THE INTERACTING WINDS MODEL}

Wallerstein's observations led to the proposal of the interacting stellar winds model for HM Sge (Kwok and Purton 1979). In this model, the permitted lines ( $\mathrm{H} \alpha$ and $\mathrm{HeI}$ ) are suggested to arise from a highspeed wind from the hot star and low-critical-density lines ([NII]) are emitted from the wind of the Mira. If the hot-star wind began at the time of the outburst, then the interaction of this wind with the preexisting Mira wind will lead to the formation of a high-density shell from which the intermediate-critical-density lines (e.g. [SIII]) are emitted. Mass of the shell will increase with time as more of the Mira wind material is swept up by the hot-star wind.

\subsection{The dynamical equations}

Assuming that the shell is made up of mostly swept-up cool-star material, mass loss from the hot star is steady, and the interaction between the hot-star wind and the shell is adiabatic, then the equations of continuity, motion and the conservation of energy can be solved by similarity analysis yielding the following solutions (Kwok 1986):

$$
\begin{aligned}
& \mathrm{R}_{\mathrm{s}}=\mathrm{V}_{\mathrm{s}} \mathrm{t} \\
& \mathrm{M}_{\mathrm{s}}=\dot{\mathrm{M}}\left(\mathrm{V}_{\mathrm{s}} / \mathrm{V}-1\right) \mathrm{t} \\
& \mathrm{P}=\frac{\frac{1}{2} \dot{\mathrm{m}} \mathrm{v}^{2}}{6 \pi \mathrm{V}_{\mathrm{s}}^{3}} \mathrm{t}^{-2}
\end{aligned}
$$

where $M_{S}$ and $V_{S}$ are the mass and velocity of the shell, $M$ and $V$ are the mass loss rate and velocity of the Mira wind, $\dot{m}$ and $v$ are the mass loss rate and velocity of the hot-star wind, and $P$ is the pressure in the shocked region which is responsible for pushing the shell. The expansion velocity $V_{s}$ is given by the root of the following equation:

$$
(\dot{\mathrm{M}} / \mathrm{V}) \mathrm{V}_{\mathrm{s}}^{3}-2 \dot{\mathrm{MV}} \mathrm{s}_{\mathrm{s}}^{2}+\dot{\mathrm{MVV}} \mathrm{s}=\frac{1}{3} \dot{\mathrm{m}} \mathrm{v}^{2}
$$

\subsection{Thermal structure of the wind interaction region}

Applying the jump conditions for a strong adiabatic shock, one finds that the location of the inner shock $\left(R_{i n}\right)$ is given by:

$$
\mathrm{R}_{\text {in }}=1.5\left(\mathrm{~V}_{\mathrm{s}} / \mathrm{v}\right)^{\frac{1}{2}} \mathrm{R}_{\mathrm{S}}
$$

For $\mathrm{V}_{\mathrm{S}}-100 \mathrm{~km} \mathrm{~s}^{-1}$ and $\mathrm{v} \sim 2000 \mathrm{~km} \mathrm{~s}^{-1}, \mathrm{R}_{\text {in }} \sim 0.34 \mathrm{R}_{\mathrm{S}}$, or approximately 968 of the volume inside $R_{S}$ is shocked. Using the ideal gas law and assuming that the shocked region is uniform in density we have 


$$
\mathrm{T}=\frac{\mu \mathrm{m}_{\mathrm{H}} \mathrm{v}^{2} \epsilon}{9 \mathrm{k}}
$$

where $\mu(\sim 0.6)$ is the mean atomic weight, $\mathrm{k}$ is Boltzmann constant, and $\epsilon$ is the filling factor. With the above parameters, the shocked region is found to have a temperature of $-10^{7} \mathrm{~K}$ (Kwok and Leahy 1984).

$\mathrm{X}$-ray emission is expected at such high temperatures and HM Sge is indeed found to be an X-ray source by the Einstein Satellite (Allen 1981). Willson et al. (1984) suggest that the X-ray emission originates from the head-on collision region of the two winds. If the interaction between the two winds is indeed adiabatic, then the analysis above suggests that the high-temperature shocked-region is not confined to a small volume but instead occupies a volume much larger than the binary separation. The analysis of the Einstein data by Kwok and Leahy (1984) finds that the observed X-ray flux is consistent with the expected emission from a shocked hot-star wind of mass loss rate of $\dot{m}-10^{-7} \mathrm{M}_{\odot}$ $\mathrm{yr}^{-1}$.

\section{OBSERVATIONAL TESTS OF THE INTERACTING WINDS MODEL}

\subsection{Radio}

The most obvious test of the predicted wind-shell structure of HM Sge is by direct imaging. Very high $(-0.08$ arc sec) resolution radio observations (Kwok, Bignell and Purton 1984) show a diffuse halo surrounding a central core of $0: 15$ in size in qualitative agreement with the mode1 prediction. A more quantitative test would be to fit the multifrequency radio light curves of Fig. 1 since measurements at different frequencies probe into different depths of the source and the optical depths are evolving with time. Using Wallerstein's (1978) velocities as input parameters, Purton, Kwok and Feldman (1983) were able to obtain reasonable fits to the light curves with the interacting winds model.

\subsection{Infrared}

While the presence of a circumstellar envelope is evident in the infrared spectrum (Fig. 1), it would be desirable to directly detect the extended far infrared emission. Figure 3 shows the $50 \mu \mathrm{m}$ map of HM Sge

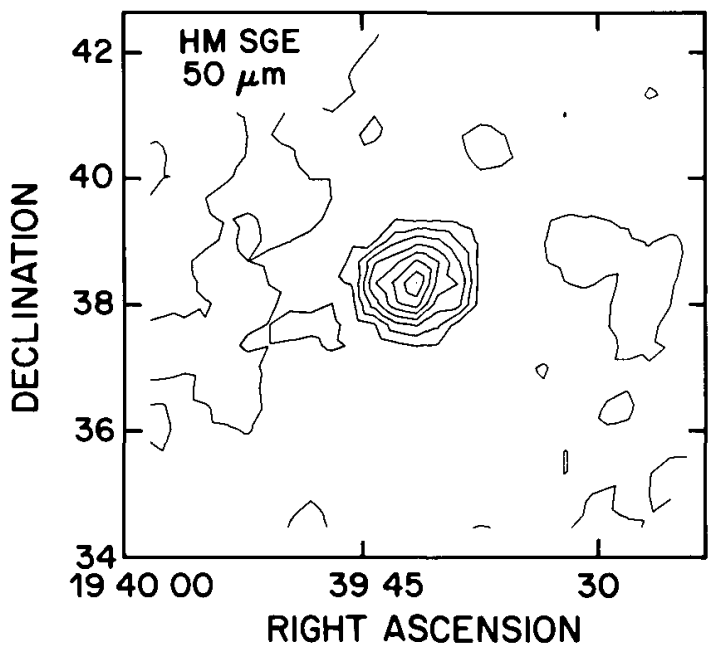

Fig. 3 IRAS CPC map of HM Sge. 
obtained by the Chopped Photometric Channel instrument on the IRAS satellite. The infrared source is not resolved at the resolution of $\sim 90$ arc sec.

\subsection{Optical}

High-resolution optical spectroscopic observations by Stauffer (1984) have found double-peak profiles in the emission lines [SIII], [NII], [OIII], etc. which suggest that these emissions originate in a shel1 expanding at $-42 \mathrm{~km} \mathrm{~s}^{-1}\left(32 \mathrm{~km} \mathrm{~s}^{-1}\right.$ in V1016 Cygni). The simultaneous presence of a narrow component (e.g. in [OI]) is interpreted as arising from the Mira wind. It is interesting to note that features with stronger narrow components have lower critical densities. In the interacting winds picture, one can then associate a densities of $10^{6.7}$ for the shell and $10^{5}$ for the halo.

\subsection{Profile asymmetries}

Asymmetric emission line profiles have been noted in V1016 Cygni and HM Sge by Solf (1983; 1984), Stauffer (1984) and Wallerstein et al. (1984). A biconical model is proposed by solf who finds that the ejections in both systems to be well collimated, with opening angles of $6^{\circ}$ and $35^{\circ}$ for HM Sge and V1016 Cygni respectively. Wallerstein et al. offer a "sphere minus cone" geometry, which could arise naturally from the collision of the two winds (Girard and Willson 1987).

\section{ENERGY SOURCES}

Figure 4 shows the energy distribution of HM Sge. It should be noted that these plotted observations were not taken at the same time, and in view of the variable nature of the object, only represent an approximate picture of the overall spectrum. Also not corrected for are the effects due to interstellar extinction. The IUE measurements are the continuum level as estimated by Feibelman (1982) and do not include the contribution from emission lines. The solid line represents the photospheric contribution as measured by Puetter et al. (1978). It is clear that most of the flux from HM Sge is emitted in the infrared, but the possibility of significant amount of flux escaping in the far ultraviolet cannot be excluded.

The distance and luminosity of HM Sge is still in controversy (Solf 1984, Kwok 1986). While the measured angular size and expansion velocity of the shell suggest a distance of $\sim 400 \mathrm{pc}$, the total observed flux and the inferred luminosity of the Mira component would put its distance $>1.4 \mathrm{kpc}$. We note that the LRS spectrum of HM Sge is among the best quality obtained by IRAS which also argues for a small distance.

\section{DURATION OF THE HOT-STAR EJECTION}

One of the assumptions used in $\S 3$ is that the system is in a steady state. However, there is evidence that the WR feature attributed to the hot-star wind has disappeared after 1980 (Feibelman 1982; Stauffer 1984) 


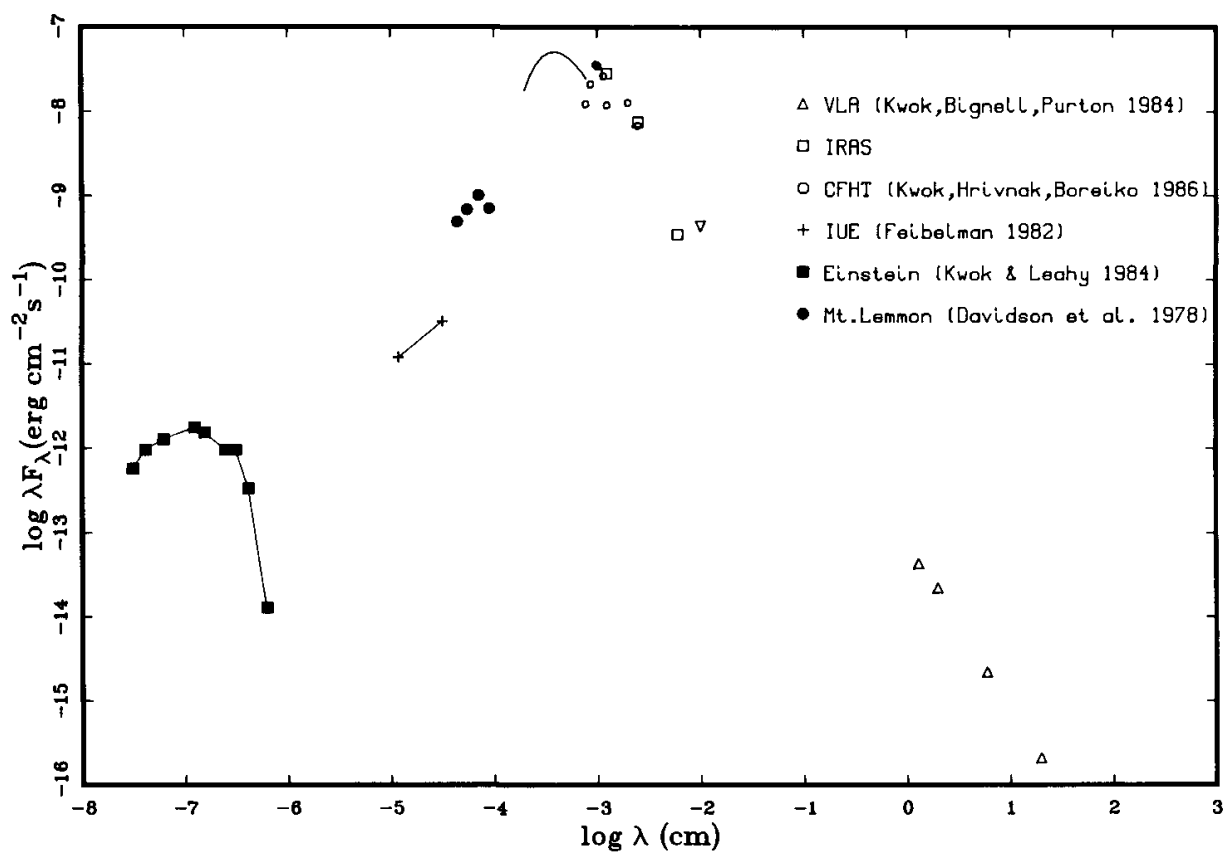

Fig. 4 Energy distribution of HM Sge.

and this may imply that the wind has been weakening with time and ceased completely in 1980. This event was accompanied by the rise in HeII 4686 line and the increase in ultraviolet fluxes of CIV, NIV, NV, etc.

(Mueller and Nussbaumer 1985). If the ejection from the hot star has indeed ceased, then the high-density shell must be decelerating and such deceleration should be detectable by continued monitoring of the radio light curves as well as by observing the profile changes of the emission lines.

The reduction in ejection rate would also cause the shrinking of the pseudo-photosphere with the effect of increasing effective temperature of the hot star. There is evidence that the excitation state of the nebula has increased after 1980, with an inferred temperature of the hot star changing from $-50,000 \mathrm{~K}$ to $160,000 \mathrm{~K}$ (Stauffer 1984).

\section{EVOLUTIONARY SCENARIO}

We can postulate the following evolutionary scenario for symbiotic novae. The two stars in the system evolve independently as if they are single stars. - Sometime after the primary has gone through the planetary nebula phase and has become a white dwarf, the secondary star evolves up the AGB and enters the Mira phase. Stellar wind from the secondary (now the more massive of the two) is accreted by the white dwarf. After enough material is accumulated, H-shell burning is ignited and a fast wind begins to flow from the white dwarf. The circumstellar material of 
the Mira acts an a barrier to this new wind and the interaction of the two results in the formation of a dense shell and a high-temperature bubble. The expansion of the shell leads to the observed radio brightening and the gradually diluting bubble is responsible for X-ray emission. It is likely that the visible brightening observed in HM Sge is not dominated by an increase in the level of continuum emission, but is the result of the dissipation of circumstellar dust and the subsequent ionization of the circumstellar envelope.

\section{CONCLUSIONS}

It has become apparent that the wind from the Mira component in symbiotic stars is responsible for the presence of nebular emission lines in many D-type symbiotics. Nova-like ejections in the hot component, even for a short period of time, introduce the possibility of colliding winds which can lead to many interesting observational consequences, including slow light curves and X-ray emissions. One may even speculate that some symbiotic stars (e.g. H1-36, AG Peg) had a history of colliding winds but have now become quiescent. This process therefore may be more common than presently realized and deserves more investigation in our quest for the understanding of the symbiotic phenomenon.

\section{REFERENCES}

Allen, D.A. 1980, Mon. Not. Roy. Astron. Soc., 912, 521. Allen, D.A. 1981, Mon. Not. Roy. Astron. Soc., 197, 739. Feibelman, W. A. 1982, Astrophys. J., $238,548$. Girard, T., and Willson, L.A. 1987, Astron. Astrophys., in press. Ipatov, A.P., Taranova, O.G., and Yudin, B.F. 1985, Astron. Astrophys., 142,85 .

Kwok, S. 1982, in The Nature of Symbiotic Stars, eds. M. Friedjung and R. Viotti, p. 209.

Kwok, S. 1986, in Highlights of Astronomy, ed. J.P. Swings, p. 189.

Kwok, S. and Purton, C.R. 1979, Astrophys. J., 229, 187.

Kwok, S. and Leahy, D.A. 1984, Astrophys. J., 283, 675.

Kwok, S., Bignell, R.C., and Purton, C.R. 1984, Astrophys. J., $279,188$. Kwok, S., Hrivnak, B.J., and Boreiko, R.T. 1986, unpublished data. Mueller, B.E.A., and Nussbaumer, H. 1985, Astron. Astrophys., 145, 144. Paczyǹski, B. 1980, Highlights of Astronomy, ed. P.A. Wayman, 5, 27. Puetter, R.C., Russe11, R.W., Soifer, B.T., and Willner, S.P. 1978 , Astrophys. J. (Letters), 223, L93.

Purton, C.R., Kwok, S., and Feldman, P.A. 1983, Astron. J., 88, 1825.

Solf, J. 1983, Astrophys. J. (Letters), 266, L113.

Solf, J. 1984, Astron. Astrophys., 139, 296.

Stauffer, J.R. 1984, Astrophys. J., 280, 695.

Volk, K., and Kwok, S. 1987, Astrophys. J., 315, 654.

Wallerstein, G. 1978, Pub1. Astron. Soc. Pacific, 90, 36.

Wallerstein, G., Willson, L.A., Salzer, J. and Brugel, E. 1984, Astron. Astrophys., 133, 137.

Willson, L.A., Wallerstein, G., Brugel, E.W., and Stencel, R.E. 1984, Astron. Astrophys., 133, 154. 